\title{
Theory, practice, and politics - Policy decision- making process based on epidemiology and public health evidence proved to be feasible in multistage research on blood-borne infections in Poland.
}

\author{
ANITA GĘBSKA-KUCZEROWSKA ( $\square$ agkucz@vp.pl ) \\ National Institute of Public Health https://orcid.org/0000-0001-9268-3731 \\ Robert Gajda \\ Gajda-Med Medical Center
}

Study Protocol

Keywords: Continuous quality improvement, Healthcare quality improvement, Health policy, Infection control, Patient safety

Posted Date: January 8th, 2020

DOI: https://doi.org/10.21203/rs.2.20327/v1

License: (c) (i) This work is licensed under a Creative Commons Attribution 4.0 International License. Read Full License 


\section{Abstract}

Objective This study presented a research methodology used to prepare the proposal of assumptions regarding the strategy for preventing blood-borne diseases in Poland.

Methods The project was carried out from 18th July to 30th November 2016. The tasks under that project were assigned to an outsourcing company to avoid the tendency in respondents' answers. The research was divided into three stages. The first stage diagnosed the problem from the perspectives of service providers, practitioners, and epidemiologists - persons who encounter problems related to blood-derivative infections at work. The second stage involved analysis at the level of local and country health policy - the institution of supervision, authorities, and administration through the engagement of experts, and public healthcare specialists. In the third stage, decision makers were interviewed (key representatives- that is, the opinions of leaders were sought) and asked to summarize and define priorities regarding conclusions determined in the earlier phases of the project, i.e., during the first and second stages.

Results The final output of the entire project is a list of key problems/challenges and solution proposals associated with medical and nonmedical services that are connected to the breakage of tissue continuity.

Conclusions Results composed from the multi-stage survey regarded proposed assumptions for the strategy for the prevention of blood-borne infections in Poland. Statistical data allowed the assessment of the health effects, while the methodology applied also allowed diagnosis of the processes leading to these effects.

Funding This study was supported by a Swiss Contribution and Ministry of Health grant (KIK35).

\section{Background}

Many researchers in public health have reported the problem of a gap between science, practice, and politics. Policymakers' decisions are a result of their goodwill, knowledge, and social and economic pressure. What is optimal is to make decisions based on scientific circumstances and facts; however, in practice, this is not always feasible. Another problem for a conscious approach to politics and decisionmaking in the field of healthcare is that the effects of actions are shifted in time, whereas costs exist as early as at the stage of preliminary analyses and for the preparation of a strategy. Therefore, exceptional arguments on the part of researchers are needed (theory and practice) to persuade brave and visionary politicians to make decisions the effects of which will be visible after their tenure ends.

Information is a crucial element in decision-making [1-3]. When program proposals are prepared, attention should also be paid to the element of participation of the people concerned with an analytical and decisionmaking process and social consultancy that facilitates the preparation of a program proposal and meets the highest expectations yet does not guarantee success. Therefore, as an argument for the opponents of 
evidence-based public health, the intention of the current authors is to present a possible method that reduces the gap between science and politics through a scientific approach to problem-solving.

Many researchers and conscious politicians believe that it is necessary to involve several stakeholders in a decision-making process $[4,5]$. Likewise, a preliminary definition of rules about the directions of reasonable decisions, related to the current knowledge about clinical sciences and public health, has also become tremendously important [6-9].

The objective of this article is to present a method for a multi-stage process of analysis using qualitative studies supported with evidence from quantitative analyses to prepare assumptions for a political strategy proposal (priority of goals). It aims to prepare a foundation for the analysis of related problems (proposal for the strategy).

\section{Materials And Methods}

This study was of a qualitative nature and was proceeded by a pilot study $[10,11]$. It was approved by the Research Board of the National Institute of Public Health. The study protocol guaranteed voluntary participation and the anonymity of all experts. Informed consent was obtained from all participants. Opinions were obtained from a broad group of experts and practitioners throughout Poland whose views might be helpful in advancing the development of a blood-borne infection prevention program. The following topics were focused on: the problem of reducing the risk of infections in services/procedures connected with abrasion and damage of tissues, and the risk associated with the spread of infections and protection of staff and clients/patients.

The data obtained were divided into four topic areas related to the following: organization and management, legislation and regulations, education, and financing. Three groups of experts were invited to participate in this research project: practitioners, employees of supervising authorities (administrative supervision) at the regional level and policy-makers at the national level (central), and opinion leaders. A qualitative study was carried out in the form of a two-panel internet discussion, and a focus group interview (FGI) was conducted with each of those groups. These tasks were outsourced to an independent research agency that performed them using an online tool (IdeaBlog).

The information collected together with supporting data (i.e., statistical figures, information on program implementations, results of supervision, control over the healthcare and nonmedical services sector, etc.) does not reflect only the status of social awareness. The substantial results of the project on blood-borne infections provided up-to-date and extensive research output material clarifying various aspects of infection risk in both healthcare and nonmedical services by considering many perspectives. Data obtained at the subsequent stages of the project represented the opinions of service providers, clients, and public 
payers of services and were used to select and identify priorities (Figure 1). This was essential in developing the proposed assumptions for the blood-borne infection prevention strategy.

\section{Procedure}

Identification of the problem

The first stage of this research project was carried out between July 18 and August 20, 2016. The following practitioners were invited during this stage: experts, epidemiologists, and service providers whose daily work is associated with the risk of blood-borne infections-both exposure to the risk and risk generation. The perspective of an authority that controls the observance of regulations regarding the minimization of that risk, i.e., Sanitary Inspectorate, was also obtained. They were invited using a list prepared by the National Institute of Public Health; the official invitation contained a presentation of the research project, its objectives, and a kind request to participate in it. Anonymity was guaranteed to all participants. Of the 80 people who were invited, 42 participated in an internet forum for five days. The main reason for refusal to participate in the study at each stage was an excessive number of other obligations, which made it impossible for them to be completely involved in the project. Throughout the study period, the invited experts were given two questions every day through which they were asked for their opinions. For each task, the necessity to support answers with evidence was emphasized; for example, from practice, figures, statistical data, and particular examples reflecting problems and good practice. The results thus collected were verified through supported material, and they constitute an extensive collection of research material that was arranged in a SWOT analysis and used in a form verified for the subsequent second stage.

Identification of the process and proposed solutions

At the second stage, the following representatives of supervising authorities participated in the study: representatives of state administration authorities, medical center managers, experts from academic centers, and specialists and experts in the field of public health. Of the 125 people invited to participate in the second stage of this research project, 41 took part in it. The research methods were similar to those used in the first stage; however, the profile of the group of experts was different. Information was gathered between September 5 and November 7, 2016. Participants received one topic per day for five days and were asked to express their opinions regarding the topics. Figures and data collected this way and verified (from the first stage and from the second stage) constituted a basis for the subsequent analysis: information selection and emergence of new aspects from the perspective of risk management. In the analysis, the obtained information was assigned to the following topics: management, legislation, education, and financing. The information was synthesized, and 33 postulates were proposed that formed the basis of discussion for the third stage of this research project. 
Priorities of tasks and problems

An FGI that summed up the first and second stages of this study was completed on November 30, 2016, at the National Institute of Public Health in Warsaw. FGIs are a qualitative method used to discuss particular themes in a group of invited participants in an open atmosphere; the proposed scenario was prepared earlier $[12,13]$. The meeting was attended by opinion leaders-representatives of patients, service providers, public payers, the Agency for Health Technology Assessment and Tarification, and the Ministry of Health. Participants did not have to represent similar standpoints; the purpose of the meeting was not to identify solutions but to understand various perspectives so that they could be considered at a later research stage. The meeting was discussion-oriented and not debate-oriented, despite the existence of many sensitive areas. There was a moderator whose role was to create conditions favorable for discussion. Since this was supposed to be a panel discussion with a group of key decision-makers to sum up the two earlier stages of this research project, based on the findings obtained in the earlier stages, 33 proposed solutions were prepared (divided into four categories: legislative, educational, organizational, and financial) and sent by email to the experts invited to the discussion before the meeting so that they could adequately prepare for the discussion. At the meeting, participants were asked to prioritize the 33 proposed solutions.

\section{Fig. 1. Project Scheme - Research Project Stages}

In the third stage, 26 postulates were adopted and prioritized according to the credits assigned to them by respondents. In this way, the problem-postulates were assigned a rank. In the first group of most important priorities, i.e., high number of credits (11-18), four postulates were included. In the second group of moderate priorities (4-7 credits), there were seven postulates. Finally, in the third group of low importance (1-3 credits), 15 postulates were included and seven were rejected. In addition, in the prioritization of tasks, like in the earlier stages of the research project, the adopted topic areas were allocated into legislation, education, organization, and finance.

\section{Discussion}

Due to the research assumptions and the practical, applicable nature of the research, a qualitative method that enables the collection of various opinions, evidence, and postulates from all stakeholders was selected. This approach is in line with the new method of managing public health proposals; qualitative methods provide participants with a space for free expression of thoughts, but they are still based on evidence. The techniques and web tools employed in qualitative research also provide a space for discussion and exchange of opinions (time for other consultations and finding evidence, and protecting anonymity) to obtain responses from participants, reflecting not only directions for their views (what they find to be positive or negative) but also substantiation of their views (why they think in that way) supported by examples/evidence. Therefore, the application of free techniques was necessary. 
Unlike standard interview techniques, these techniques enable a flexible reaction to participants' responses and the expansion of resultant information. In the field of public health, qualitative techniques comprise an additional, valuable source of information that enables the rationalization of a decision-making process, provides an opportunity to take a broader view of the problem, and includes other stakeholders in the interpretation and decision-making process [14-18]. The online platform IdeaBlog, proposed by a research company, turned out to be a good research tool. The evaluation of reliability/quality of analyses is usually based on data from quantitative research. For example, known methodologies such as GRADE and AGREE are largely related to the implementation of results from other studies into policy [19-22]. In the analysis of data, especially in relation to the assessment of efficacy and safety of treatment, these studies are of indisputable importance. Relatively less importance has been attached to qualitative studies, which also require different methodologies and indications for their application. An ideal solution is to reach a consensus and apply analyses combining simultaneous analyses of qualitative and quantitative studies in the development of recommendations, as shown by the fact that the analyses of these approaches are differentiated.

The methodology of the multi-stage process of research and analysis presented in this article combines the approach of qualitative data research with selective analysis of quantitative data (statistical data, research results and expert opinions, case studies, publications, etc.), which were a condition of the first and second stages. The project included a sequence of diagnosis of the situation (results from the first stage) and diagnosis of decision-making processes (results of the second stage), and a discussion on a coherent catalogue of the developed data of the first and second stages (results of the third stage). At each stage of the study, there were references to the practical links between theory and practice, both at the stage of problem diagnosis and at that of programs/procedures. This research approach is consistent with the opinions of many other researchers and includes both the creation of recommendations and their updating based on data.

The present analysis, divided into status diagnosis and decision-making processes, was an attempt to systemize information about the extent of risk associated with blood-borne infections, and the essential stages of analysis for the preparation of further stages-proposed solutions. This is consistent with a methodological "mixed research approach" represented by other researchers in the field of public health and healthcare policy [23-27]. The first and second stages of this research project were carried out using forum methodology/discussion (IdeaBlog). No quantitative methods were employed since the objective of this study was not to measure the scale (number) of a phenomenon but the extent to which the identified problems and opinions are repetitive in the group of participating experts. However, respondents were asked for their opinions with supporting evidence in the form of statistical data and other sources. First, the present researchers collected qualitative data and figures and diagnosed the status of knowledge (practical and theoretical) and the functioning of the system of services with regard to a problem researched. The research web tool provided the possibility of ensuring respondents' anonymity and respecting their preferences concerning the time and place of the interviews. The anonymous interviews were supervised. An additional advantage of the website interview-research blog was the possibility of mutual discussion between respondents on selected topics and adequate time for collecting evidence for external 
consultations. In the final stage, collected data on the diagnosis of the system and assessment of the scale of the risk of blood-borne infections was systematized, and priorities were identified through ranking. In terms of public health evidence, the results of this research project, in the form of extensive reports from each research stage, were submitted to the Hepatitis C-virus Project Board to propose a new strategy. These reports were also presented to all stakeholders as a source of information on the issue of blood-borne diseases in Poland. The selection of solutions resulted from substantive reasons (i.e., the intention to collect possibly the most extensive material about the foundations and the actions taken by various institutions in the context of the prevention of blood-borne infections) and organizational and technical reasons (since the target group covered and represented various regions in Poland, as well as people with different professional backgrounds and responsibilities).

\section{Summary And Conclusion}

A three-stage research approach based on results of qualitative and quantitative research evidence is presented. In the first stage, the risk of infection in services was assessed. In the second stage, infection risk issues related to processes and procedures were evaluated. In the third stage, decision-makers were asked to summarize and prioritize the lessons learned from the earlier phases of the project. The impartiality of the investigation was guaranteed by the independent research organization carrying out the surveys. Additionally, new techniques were applied that guaranteed anonymity according to the project protocol. Economic realities and the dynamics of changes in the field of epidemiology as well as pressure from patients/clients/service providers, who are aware of the risk, provide the possibility of numerous decisions. This is a privilege of democracy, but it also increases the obligation of experts to prepare recommendations to make research tools reliable and credible and to give broader insight into the problem being studied.

This research project also has a limitation, which is important to note here. The detailed analysis did not consider current regulations or organizational and financing structure; instead, it only indirectly referred to the opinions of experts and the information provided by them. A substantial research limitation was resignation of invited experts from full-time participation due to their professional duties. Some experts refused due to doubts concerning the possibility of political persuasion and limitations in the implementation of prepared proposals. In addition, the system of motivating participation in the survey was insufficient, although it was voluntary and not accompanied by any financial compensation.

Nevertheless, the findings of the present study are significant and provide directions for further research. In the future, the current authors will verify the extent to which the present results contributed to improvements in the field-for example, health condition in terms of blood-borne infections-and whether the presented method of priority selection will find supporters (social consultations and acceptance).

\section{Key messages}


1. Evidence-based public health is routine. There is also an ongoing need to seek methods for evaluating the effects and processes of action taken in management in public health. A standard sentinel system and epidemiological research provide strong statistical evidence and are the basis for rational decision-making. Improvement of the quality of statistical data and the need to broaden knowledge with new and better diagnostic tools inspire the search for better qualitative and quantitative methods in public health. This is particularly important in areas of public health in which there are similar risks and different approaches in terms of prevention as well as different legal responsibilities.

Another challenge was to look at the problem of blood-borne diseases from the perspectives of medical and non-medical services, and the application of the presented methodology was designed to help answer the question of whether a more careful approach to the prevention of blood-borne infections can be observed in the medical services sector. It is possible to implement such an approach in the non-medical services sector and to more closely examine the systems of medical services for indications of areas where the functioning needs improvement in order to guarantee greater safety (prevention) and adequate disruption of the transmission chain of infections.

1. The methodology applied in this study allowed for a detailed analysis of the problem of infections in services related to tissue discontinuity. The combination of qualitative and quantitative methodologies and an attempt to find optimal solutions were assumed to be useful for the development of a strategy for the prevention of blood-borne infections. The methodology applied and the results obtained also inspired changes in the education programs for employees in both the medical and non-medical service sectors in terms of the prevention of blood-borne infections. This is allowed the consolidation of various professional and social environments in order to reach a common consensus regarding the development of an infection risk reduction program.

From the perspective of public health, it is important to understand the limitations and capacity for action, as well as to maintain updated information on risk.

1. The report from the tri-stage analysis was presented for decision-makers at various levels of management (in the medical and non-medical service sectors). The proposal for a health policy prevention strategy for blood-borne infections was then prepared with input from the research. The policy included extension of education programs based on analysis and educational material in direct e-learning. Additionally, specialized training programs for nurses were amended, indicating the need to modify the training programs for other professional groups.

\section{Abbreviations}

SWOT - Strengths, Weaknesses, Opportunities, Threats

FGI - focused group interview 
KIK35 - project finance acronym

GRADE - Grading Recommendations Assessment and Development Evidence

AGREE - Appraisal of Guidelines for Research \& Evaluation

\section{Declarations}

\section{Ethics approval and consent to participate}

Not applicable

\section{Consent for publication}

Not applicable

\section{Availability of data and materials}

The corresponding author had full access to all the data in the study and had final responsibility for the decision to submit for publication.

\section{Competing interests}

The authors declare that they have no competing interests.

\section{Funding}

The Study was supported by a Swiss Contribution and Ministry of Health grant (KIK35). The tasks under that project were assigned to an outsourcing company (financed) to avoid conflict of interests. The funders of the study had no role in study design, data collection, data analysis, data interpretation, or writing of the report. The outsourced independent research agency performed research according to project protocol (presented methodology). At each stage, research workers monitored the course of anonymous interviews and discussion moderation, restructuring it depending on the necessity to focus discussion on particular problems.

\section{Authors' contributions}

All authors have read and approved the manuscript. The corresponding author had full access to all data required in the process of this research and has finally assumed the responsibility for the decision to submit this paper for publication. AGK was responsible for: study design (PI), literature search, figures, data analysis, data interpretation, and writing. RG was responsible for: literature search, data analysis, data interpretation, and writing.

\section{Acknowledgments}


We thank the sponsors of the research for their financial support. The authors thank persons from Kantar Mill Word Brown for their professional cooperation in this research, especially Dr. Magdalena Stec, experienced interviewer and facilitator, for her extraordinary merit-based support. We sincerely thank our invited experts and specialists for their availability, time, and contributions. We thank the Public Health and Epidemiology Consultants for their support. We are very grateful to Mr. Grzegorz Swięćkowski for reviewing the bibliography. We give special thanks to EDITAGE by Cactus for professional help in English editing of the article.

\section{References}

1. Sorian R, Baugh T. Power of information: Closing the gap between research and policy. Health Affairs. 2002;21(2):264-273 DOI: https://doi.org/10.1377/hlthaff.21.2.264

2. Lomas J. Decision support: A new approach to making the best healthcare management and policy choices. Healthcare Quarterly 2007;10:16-8.

3. Oderkirk J, Sassi F, Cecchini M, et al. Toward a New Comprehensive International Health and Health Care Policy Decision Support Tool. OECD Health Division 2012.

https://ec.europa.eu/health//sites/health/files/projects/docs/2010_health_healthcare_policy_fr_en.pdf

4. Abelson J, Forest PG, Eyles J, et al. Examining the role of context in the implementation of a deliberative public participation experiment: Results from a Canadian comparative study. Soc Sci Med. 2007;64(10):2115-28.

5. Abelson J, Forest PG, Eyles J, et al. Deliberations about deliberative methods: Issues in the design and evaluation of public participation processes. Soc Sci Med 2003;57(2):239-51.

6. Black N, Murphy M, Lamping D, et al: Consensus development methods: A review of best practice in creating clinical guidelines. J Health Serv Res Policy 1999;4(4):236-48.

7. Lavis JN, Boyko JA, Oxman AD, et al. SUPPORT Tools for evidence-informed health Policymaking (STP). 13. Preparing and using policy briefs to support evidence-informed policymaking. Health Res Policy Syst 2009;7(Suppl 1):S13. DOI: https://doi.org/10.1186/1478-4505-7-S1-S13.

8. Lavis JN, Oxman AD, Lewin S, et al.: SUPPORT Tools for evidence-informed health policymaking (STP). Introduction. Health Res Policy Syst 2009;7(Suppl 1):I1. DOI: https://doi.org/10.1186/1478-4505-7-S111.

9. Lavis JN, Permanand G, Oxman AD, et al.: SUPPORT Tools for evidence-informed health policymaking (STP). 13. Preparing and using policy briefs to support evidence- informed policymaking. Health Res Policy Syst 2009;7(Suppl 1):S13. DOI: 10.1186/1478-4505-7-S1-S13.

10. Silverman D. Qualitative Research. $3^{\text {rd }}$ ed. London: Sage Publishing 2016.

11. Leary MR. Introduction to Behavioral Research Methods. 4th ed. Auckland, New Zealand: Pearson Education New Zealand 2014.

12. Greenbaum TL. The Handbook for Focus Group Research. $2^{\text {nd }}$ ed. Sage Publishing 1997. 
13. Stewart DW, Shamdasani PM. Focus Groups. Theory and Practice. $3^{\text {rd }}$ ed. Sage Publishing 2015.

14. Femdal I, Solbjør M. Equality and differences: group interaction in mixed focus groups of users and professionals discussing power. Society, Health \& Vulnerability 2018;9(1):1447193. DOI: 10.1080/20021518.2018.1447193.

15. Berg BL. Qualitative Research Methods for the Social Sciences. $5^{\text {th }}$ ed. Pearson 2004.

16. Denzin NK, Lincoln YS. Handbook of Qualitative Research. $4^{\text {th }}$ ed. Sage Publishing 2011.

17. Shaughnessy JJ, Zechmeister EB, Zechmeister JS. Research Methods in Psychology. 5th ed. New York, NY, US: McGraw-Hill 2000.

18. WHO. Policy dialogue preparation and facilitation checklist, EVIPNet Europe. 2016. http://www.euro.who.int/_data/assets/pdf_file/0017/323153/EVIPNET-PD-preparation-facilitationchecklist.pdf (accessed 7 Apr 2017)

19. Welch VA, AkI EA, Guyatt G, et al. GRADE equity guidelines 1: considering health equity in GRADE guideline development: introduction and rationale. J Clin Epidemiol 2017;90:59-67 DOI: https://doi.org/10.1016/j.jclinepi (access 2017.01.014)

20. Akl EA, Welch V, Pottie $K$, et al. GRADE equity guidelines 2: considering health equity in GRADE guideline development: equity extension of the guideline development checklist. Journal of Clinical Epidemiology 2017;90:68-75 DOI: https://doi.org/10.1016/j.jclinepi.2017.01.017

21. O'Sullivan JW, Albasri A, Koshiaris C, et al. Diagnostic test guidelines based on high-quality evidence had greater rates of adherence: a meta-epidemiological study. Journal of Clinical Epidemiology 2018;103:40-50 DOI: https://doi.org/10.1016/j.jclinepi.2018.06.013

22. Welch VA, AkI EA, Pottie K, et al. GRADE equity guidelines 3: considering health equity in GRADE guideline development: rating the certainty of synthesized evidence. J Clin Epidemiol 2017;90:76-83 DOI: 10.1016/j.jclinepi.2017.01.015

23. Gopinathan U, Hoffman SJ. Institutionalizing an evidence-informed approach to guideline development: progress and challenges at the World Health Organization. BMJ Global Health 2018;3:e000716.

24. Mustafa RA, Wiercioch W, Arevalo-Rodriguez I, et al. Decision making about healthcare-related tests and diagnostic test strategies. Paper 4: International guidelines show variability in their approaches. Journal of Clinical Epidemiology 2017;82:38-46 DOI: https://doi.org/10.1016/j.jclinepi.2017.09.004

25. Straus SE, Tetroe JM, Graham ID. Knowledge translation is the use of knowledge in health care decision making. Journal of Clinical Epidemiology 2011;64(1):6-10 DOI: https://doi.org/10.1016/j.jclinepi.2009.08.016

26. Kastner M, Bhattacharyya $\mathrm{O}$, Hayden L, et al. Guideline uptake is influenced by six implementability domains for creating and communicating guidelines: a realist review. Journal of Clinical Epidemiology 2015;68(5):498-509 DOI: https://doi.org/10.1016/j.jclinepi.2014.12.013

27. Agbassi C, Messersmith $\mathrm{H}, \mathrm{Mc}$ Nair S, et al. Priority-based initiative for updating existing evidencebased clinical practice guidelines: the results of two iterations. Journal of Clinical Epidemiology 2014;67(12):1335-1342 DOI: https://doi.org/10.1016/j.jclinepi.2014.06.013

Page $11 / 12$ 


\section{Figures}

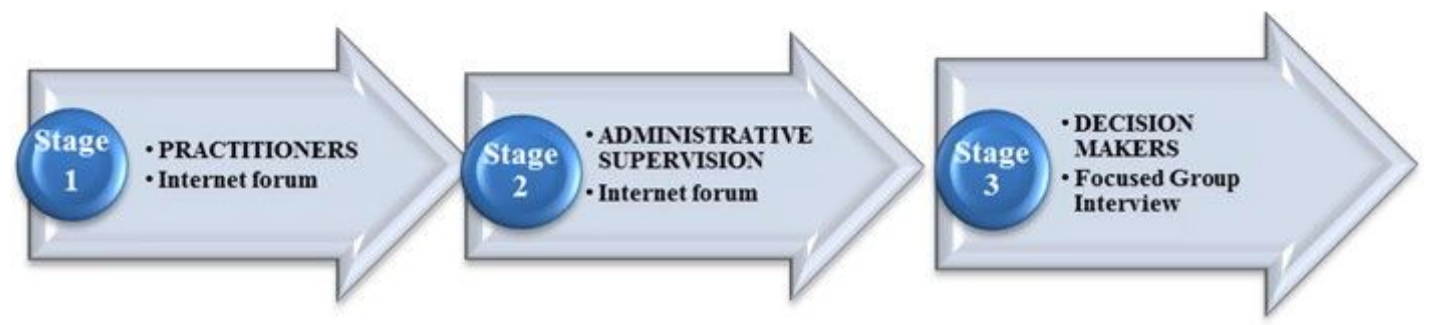

Figure 1

Scheme - Project Stages (target groups, methods) 